\title{
Early childhood adversities and risk of eating disorders in women: A Danish register-based cohort study
}

\author{
Janne Tidselbak Larsen MSc1,2,3 (D) | Trine Munk-Olsen PhD1,2,3 | \\ Cynthia M. Bulik PhD $4,5,6$ ～Laura M. Thornton PhD4 | Susanne Vinkel Koch PhD7,8 | \\ Preben Bo Mortensen DrMedSc1,2,3 | Liselotte Petersen PhD1,2,3
}

${ }^{1}$ National Centre for Register-based Research, Aarhus BSS, Aarhus University, Aarhus, Denmark

${ }^{2}$ Lundbeck Foundation Initiative for Integrative Psychiatric Research (iPSYCH), Aarhus University, Aarhus, Denmark

${ }^{3}$ Centre for Integrated Register-based Research (CIRRAU), Aarhus University, Aarhus, Denmark

${ }^{4}$ Department of Psychiatry, University of North Carolina at Chapel Hill, Chapel Hill, North Carolina

${ }^{5}$ Department of Nutrition, University of North Carolina at Chapel Hill, Chapel Hill, North Carolina

${ }^{6}$ Department of Medical Epidemiology and Biostatistics, Karolinska Institutet,

Stockholm, Sweden

${ }^{7}$ Mental Health Centre for Child and Adolescent Psychiatry, Copenhagen Region, Copenhagen, Denmark

${ }^{8}$ Institute of Clinical Medicine, Faculty of Health Sciences, University of Copenhagen, Copenhagen, Denmark

\section{Correspondence}

Janne Tidselbak Larsen, MSc, National

Centre for Register-based Research, Aarhus BSS, Aarhus University, Fuglesangs Allé 26, 8210 Aarhus V, Denmark.

Email: jtl@econ.au.dk

Funding information

The Anorexia Nervosa Genetics Initiative (ANGI), an initiative of the Klarman Family Foundation, Grant Number: VR Dnr: 5382013-8864

\begin{abstract}
Objective: Previous studies evaluating the association between early childhood adversities and eating disorders have yielded conflicting results. The aim of this study is to examine the association between a range of adversities and risk of anorexia nervosa (AN), bulimia nervosa (BN), and eating disorder not otherwise specified (EDNOS) in 495,244 women.
\end{abstract}

Method: In this nationwide, register-based cohort study, nine types of early childhood adversity (family disruption, residential instability, placement in out-of-home care, familial death, parental somatic illness, parental psychiatric illness, parental disability, severe parental criminality, and parental substance use disorder) were defined and exposure during the first 6 years of life was determined. Hazard ratios for eating disorders were calculated using Cox regression.

Results: Few adversities were significantly associated with AN, and for each, the presence of the adversity was associated with lower risk for AN. BN, and EDNOS were positively associated with several types of adversities. AN rates were unchanged or reduced by up to $54 \%$ by adversities, whereas rates of BN and EDNOS were unchanged or increased by adversities by up to 49 and $89 \%$, respectively.

Discussion: Our findings indicate that childhood adversities appear to be associated with an increased risk of BN and in particular EDNOS, whereas they seem to be either unassociated or associated with a decreased risk of AN.

\section{KEYWORDS}

anorexia nervosa, bulimia nervosa, early childhood adversity, eating disorders, eating disorder not otherwise specified, epidemiology

\section{1 | INTRODUCTION}

Eating disorders are severe illnesses associated with elevated mortality (Arcelus, Mitchell, Wales, \& Nielsen, 2011; Zerwas et al., 2015), suicidal behavior (Zerwas et al., 2015), and other adverse outcomes (Field et al.,
2012). Various psychiatric disorders have been shown to be associated with adversities in childhood (Björkenstam, Burström, Vinnerljung, \& Kosidou, 2016; Dahl et al., 2017; Laursen, Munk-Olsen, Nordentoft, \& Mortensen, 2007; Mok, Webb, Appleby, \& Pedersen, 2016). However, previous studies evaluating the association between early adversities 
and anorexia nervosa (AN) and bulimia nervosa (BN) have yielded conflicting results, and for eating disorder not otherwise specified (EDNOS) little research has been conducted. Parental psychiatric illness and family disruption have been reported to increase the risk of both AN (Fairburn, Cooper, Doll, \& Welch, 1999) and BN (Boumann \& Yates, 1994; Webster \& Palmer, 2000). Residential changes and high parental educational level are associated with increased risks of both AN (Ahrén, Chiesa, Klinteberg, \& Koupil, 2012; Fairburn et al., 1999) and of eating disorders in general (Ahrén et al., 2013; Mok et al., 2016).

Parental death before age 10 has been associated with increased risk of BN (Su et al., 2016). A dose-response pattern with increasing risk of AN for higher number of adversity factors has been found (Fairburn et al., 1999), and household living on public assistance during child ages $0-15$ years has been associated with decreased risk of pooled eating disorders (Björkenstam et al., 2016).

To expand the existing knowledge base and to evaluate to what extent early adversities influence the risk of developing various eating disorders, we examined the association between a range of measures of early childhood adversity and later risk of AN, BN, and EDNOS separately. Furthermore, we examined the effect of multiple adversities and compared the risk estimates for eating disorders to those for other psychiatric disorders with overlapping symptomatology and traits, that is, major depressive disorder (MDD), anxiety disorder, and obsessive compulsive disorder (OCD). The selection of included adversities was informed by a previous publication (Dahl et al., 2017), which examined the same panel of adversities in severe unipolar depressive disorder.

\section{2 | METHOD}

\subsection{Study population}

Using Danish national registers, we conducted a population-based cohort study including all females born in Denmark to Danish-born parents between January 1, 1989 and December 31, 2007. Individuals who died or emigrated before age 6 were excluded from the study population, resulting in a study population of 495,244 women.

Identification of cohort members was possible by using the Central Person Register number, a unique personal identification number assigned to all Danish citizens (Pedersen, 2011). This is used as identifier in all national registers and enables accurate linkage between registers.

Neither patient informed consent nor approval from an ethics committee is required for registry-based studies in Denmark.

\section{2 | Eating disorders and other psychiatric disorders}

Information on contacts to Danish hospitals are registered in The Danish National Patient Register (NPR) (Lynge, Sandegaard, \& Rebolj, 2011) or The Danish Psychiatric Central Research Register (PCRR) (Mors, Perto, \& Mortensen, 2011). From 1994 and onwards, the diagnostic system has been the International Classification of Diseases, 10th revision (ICD-10).

The events of interest were an ICD-10 diagnosis of AN (F50.0, F50.1), BN (F50.2, F50.3), or EDNOS (F50.8, F50.9).
Date of onset was defined as the admission date of the first in- or outpatient contact after the 6th birthday (chosen to reduce diagnostic misclassification, for example, feeding disorders in infancy and childhood) leading to a discharge diagnosis of the disorder of interest.

To compare the magnitude of the possible associations between childhood adversities and eating disorders to those for other psychiatric disorders in the same setting and cohort, we conducted parallel analyses for MDD (ICD-10: F32, F33), anxiety disorder (F40, F41), and OCD (F42).

\section{3 | Early childhood adversities}

We investigated nine different adversities and each of these was defined dichotomously depending on whether the child had been exposed at least once during the first 6 years of life, that is, between ages 0 and 5 years. Information on parental adversities was obtained by identifying parents of each person in the cohort using the Danish Civil Registration System (CRS) (Pedersen, 2011) and linking to information from various other registers.

Family disruption was defined as the child not sharing her address with both legal parents, based on information from Statistics Denmark. Instances when family disruption was caused by death or imprisonment of a parent or the child being placed in out-of-home care $(\mathrm{OOHC})$ were included as separate adversities and therefore not included as family disruptions.

Parental somatic illness was defined as at least one parent with a record in the NPR of having received a diagnosis of any of the severe and chronic illnesses encompassed in the Charlson Comorbidity Index (CCI) (Thygesen, Christiansen, Christensen, Lash, \& Sørensen, 2011). For this study, dementia diagnoses were omitted from the $\mathrm{CCl}$ as they were included in the measure for parental psychiatric illness.

Residential instability was defined as the child having moved between two Danish municipalities more than once, based on residence information.

Parental psychiatric illness was defined as at least one parent receiving any psychiatric diagnosis [International Classification of Diseases, 8th revision (ICD-8): 290-315 for events prior to 1994; ICD-10: F00-F99 for events from 1994 onward] with the exception of substance use disorder (SUD) in the PCRR. SUD is included as a separate exposure variable.

Severe parental criminality was defined as at least one parent having been convicted of a criminal offense under the penal law, special legislation regarding drugs and weapons, or sections of the traffic act regarding impaired driving. Only severe offenses resulting in custodial sentences were included. Information on criminal transgressions and convictions is registered in The Danish National Crime Register (Kyvsgaard, 2002).

Parental disability was considered present if the primary occupation of at least one parent was recorded in the Integrated Database for Labor Market Research (Petersson, Baadsgaard, \& Thygesen, 2011) as disability pensioner.

Familial death was defined as the child having lost a parent or a full or half sibling, based on information from the CRS. 
Parental SUD was defined as at least one parent having received an SUD diagnosis in the PCRR (ICD-8: 291.x9, 303.x9, 303.20, 303.28, 303.90, 304.x9, ICD-10: F10-F19).

Placement in $\mathrm{OOHC}$ was defined as the child having been placed in a foster home or institution, with or without parental consent, based on information from The Register for Support for Children and Adolescents.

We furthermore included dichotomous measures for any adversity (at least one of the included adversities), any maternal and any paternal adversity (at least one of maternal/paternal somatic illness, psychiatric illness, severe criminality, disability, death, or SUD), and number of different adversities.

We originally intended to include childhood abuse (combined sexual, emotional, and physical abuse captured by contacts to hospitals) in this study. Unfortunately, after exploration of the prevalence of the events, we found that we had insufficient data to include this severe adversity and still comply with Danish legislation regarding personally identifiable information.

\subsection{Statistical analysis}

Survival analyses were conducted using Cox proportional hazards models and the resulting estimates were hazard ratios (HRs) with $95 \%$ confidence intervals $(\mathrm{Cls})$. A HR of 1.00 indicates that there is no association between exposure and outcome. Each person in the study population was followed from their 6th birthday and until their first diagnosis of interest after age 6, date of death or emigration from Denmark, or December 31, 2013, whichever occurred first (i.e., until age 25 at most). The reference groups in all analyses were persons with no registered adversities during early childhood. All estimates were adjusted for age in the nonparametric part of the model and for timevarying calendar-time, divided into 5-year intervals starting in 1995 (model 1). In two extended models, we further adjusted for possible confounders by including time-varying history of psychiatric comorbidity (any psychiatric illness excluding any eating disorder) (model 2), or urbanicity at birth (categorized in five groups depending on the population density of the municipality where the child was born), parental educational level the year of the child's 6th birthday [dichotomously defined as either both parents having low educational attainment (basic schooling, vocational training, or high school) or at least one parent having high educational attainment (short, medium, or long-term higher education)], and time-varying history of parental psychiatric illness (defined as at least one parent having received any psychiatric diagnosis) (model 3). For the last model, the adversities parental psychiatric illness and parental SUD were not included as exposures and were left out of the measure for any adversity and the number of adversities. Level of urbanicity at birth is a risk factor for being diagnosed with psychiatric illness in general and with eating disorders and AN in particular (Vassos, Agerbo, Mors, \& Pedersen, 2016) and adjusting for urbanicity takes into account possible selection bias due to better access to hospital care in urban areas and thereby higher probability of having a psychiatric diagnosis in the registers. Similarly, parental educational levels
(Ahrén et al., 2012) and parental psychiatric illnesses (Fairburn et al., 1999) are risk factors for eating disorders in the offspring.

We further conducted sensitivity analyses, where only the first eating disorder diagnosis received was used in the definition of cases, that is, an individual was only considered an AN case if this was her first eating disorder diagnosis and she had not previously been diagnosed with BN or EDNOS. In the rare event of multiple eating disorder diagnoses on the same date, we let AN trump BN and EDNOS, and BN trump EDNOS.

We tested for a linear trend for the associations between number of adversities and risk of eating disorders in model 1 using likelihoodratio tests. Cumulative incidences of $\mathrm{AN}, \mathrm{BN}$, and EDNOS by number of adversities were calculated using competing-risks regression with death as the competing event.

All analyses were conducted using Stata version 13.

\section{3 | RESULTS}

Our study population was followed for a combined total of 4.8 million person-years, during which 2,892 women $(0.58 \%$ of the study population) were diagnosed with $\mathrm{AN}, 1,027(0.21 \%)$ with $\mathrm{BN}$, and 2,150 (0.43\%) with EDNOS. Approximately one-third of all three eating disorder groups were diagnosed with at least one of the three comparison disorders MDD, anxiety disorder, or OCD during follow-up.

During the first 6 years of life, more than a third of the population (34.77\%) had been exposed to any adversity, with the most common adversity being family disruption (21.74\%) and the least common placement in $\mathrm{OOHC}$ (0.67\%); see Table 1 for details (Supporting Information Table S1 for comparison disorders MDD, anxiety disorder, and OCD).

The mean number of adversities experienced by the cohort and by each case group is presented in Figure 1. AN stands out as the disorder with the lowest mean number of adversities.

\subsection{Association between early childhood adversities and risk of eating disorders}

In Table 2 estimates for eating disorders are presented. The corresponding results for comparison disorders are presented in Supporting Information Table S2.

\subsection{1 | Anorexia nervosa}

Severe parental criminality, placement in $\mathrm{OOHC}$, and any paternal adversity were all significantly associated with decreased risk of AN in model 1 with reductions in rates of $\mathrm{AN}$ of up to $54 \%$, observed for placement in $\mathrm{OOHC}$. These all remained significant in the extended models. In model 2, the estimates were in fact further decreased, and family disruption and any adversity were also significantly associated with decreased risk of AN. Indication of a decreasing linear trend was evident, although nonsignificant [change in HR per adversity: 0.95 (0.91-1.00), data not shown]. 
TABLE 1 Number of cohort members and cases exposed to early childhood adversities.

\begin{tabular}{|c|c|c|c|c|}
\hline & $\begin{array}{l}\text { Cohort } \\
(N=495,244) \\
N(\%)\end{array}$ & $\begin{array}{l}\text { AN cases } \\
(N=2,892) \\
N(\%)\end{array}$ & $\begin{array}{l}\text { BN cases } \\
(N=1,027) \\
N(\%)\end{array}$ & $\begin{array}{l}\text { EDNOS cases } \\
(N=2,150) \\
N(\%)\end{array}$ \\
\hline Family disruption & $107,672(21.74)$ & $622(21.51)$ & $285(27.75)$ & $563(26.19)$ \\
\hline Parental somatic illness & $41,776(8.44)$ & 205 (7.09) & $69(6.72)$ & $181(8.42)$ \\
\hline Residential instability & $37,613(7.59)$ & $213(7.37)$ & $86(8.37)$ & $191(8.88)$ \\
\hline Parental psychiatric illness & $22,756(4.59)$ & $113(3.91)$ & $43(4.19)$ & $130(6.05)$ \\
\hline Severe parental criminality & $10,384(2.10)$ & $42(1.45)$ & $19(1.85)$ & $58(2.70)$ \\
\hline Parental disability & $9,016(1.82)$ & 40 (1.38) & $7(0.68)$ & $50(2.33)$ \\
\hline Familial death & $4,926(0.99)$ & $36(1.24)$ & $15(1.46)$ & $26(1.21)$ \\
\hline Parental SUD & $4,540(0.92)$ & $24(0.83)$ & $12(1.17)$ & $26(1.21)$ \\
\hline Placement in $\mathrm{OOHC}$ & $3,330(0.67)$ & $10(0.35)$ & $6(0.58)$ & $19(0.88)$ \\
\hline Any adversity & $172,173(34.77)$ & $976(33.75)$ & $400(38.95)$ & $870(40.47)$ \\
\hline Any maternal adversity & $39,380(7.95)$ & $206(7.12)$ & $66(6.43)$ & $208(9.67)$ \\
\hline Any paternal adversity & $40,260(8.13)$ & $194(6.71)$ & $72(7.01)$ & $184(8.56)$ \\
\hline \multicolumn{5}{|l|}{ Number of adversities } \\
\hline 0 & $323,071(65.23)$ & $1,916(66.25)$ & $627(61.05)$ & $1,280(59.53)$ \\
\hline 1 & $121,947(24.62)$ & 727 (25.14) & 299 (29.11) & $609(28.33)$ \\
\hline 2 & $36,453(7.36)$ & $192(6.64)$ & $72(7.01)$ & $185(8.60)$ \\
\hline 3 or more & $13,773(2.78)$ & $57(1.97)$ & $29(2.82)$ & $76(3.53)$ \\
\hline
\end{tabular}

Note: $\mathrm{AN}=$ anorexia nervosa; $\mathrm{BN}=$ bulimia nervosa; $\mathrm{EDNOS}=$ eating disorder not otherwise specified; $\mathrm{SUD}=$ substance use disorder; $\mathrm{OOHC}=$ out-ofhome care.

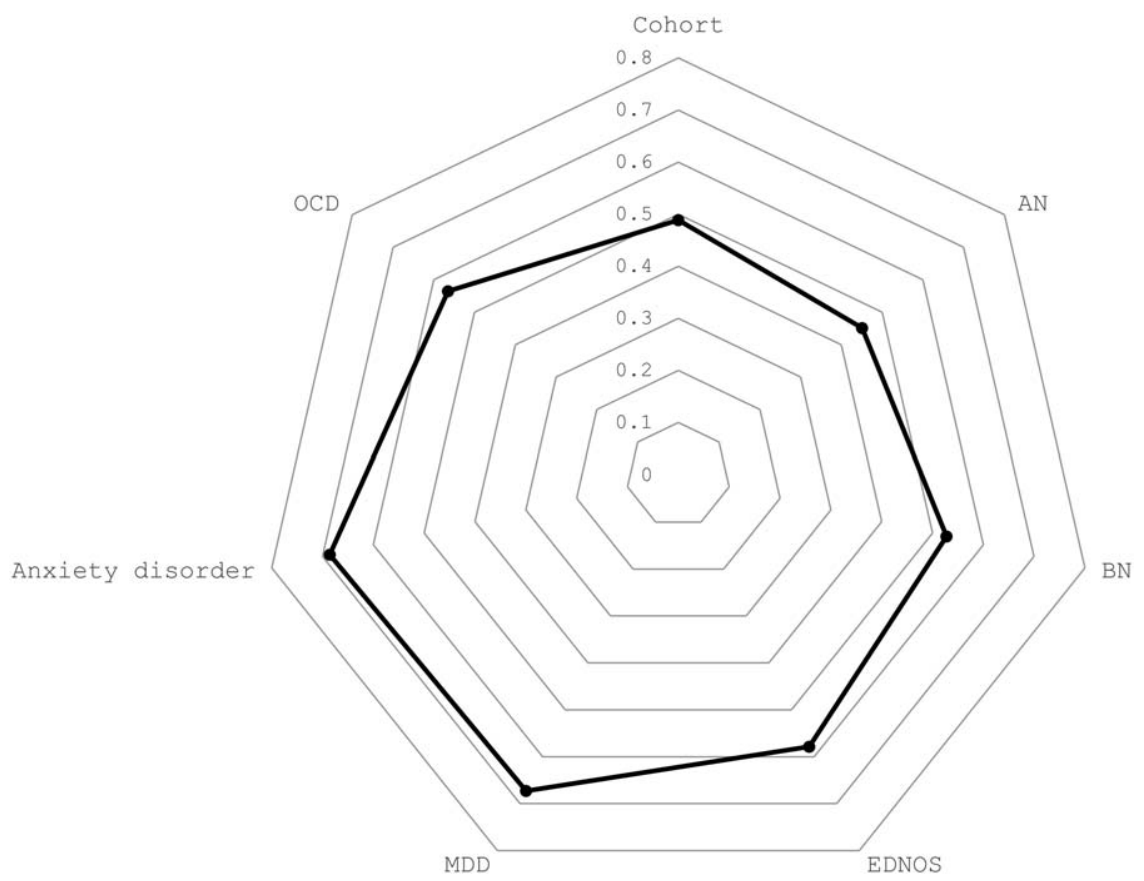

FIGURE 1 Mean number of adversities experienced between ages 0-5 years by the entire cohort and by case groups. The radar chart depicts the mean number of adversities by diagnostic group. The bolded line and the position of the data points on the spokes, reflects the mean number of adversities with points farthest from the center representing higher mean number of adversities and points closer to the center representing fewer mean numbers of adversities. Spokes represent the entire cohort and the separate diagnostic groups: $\mathrm{AN}$ = anorexia nervosa; $\mathrm{BN}$ = bulimia nervosa; $\mathrm{EDNOS}$ = eating disorder not otherwise specified; $\mathrm{MDD}=$ major depressive disorder; $\mathrm{OCD}=$ obsessive compulsive disorder 


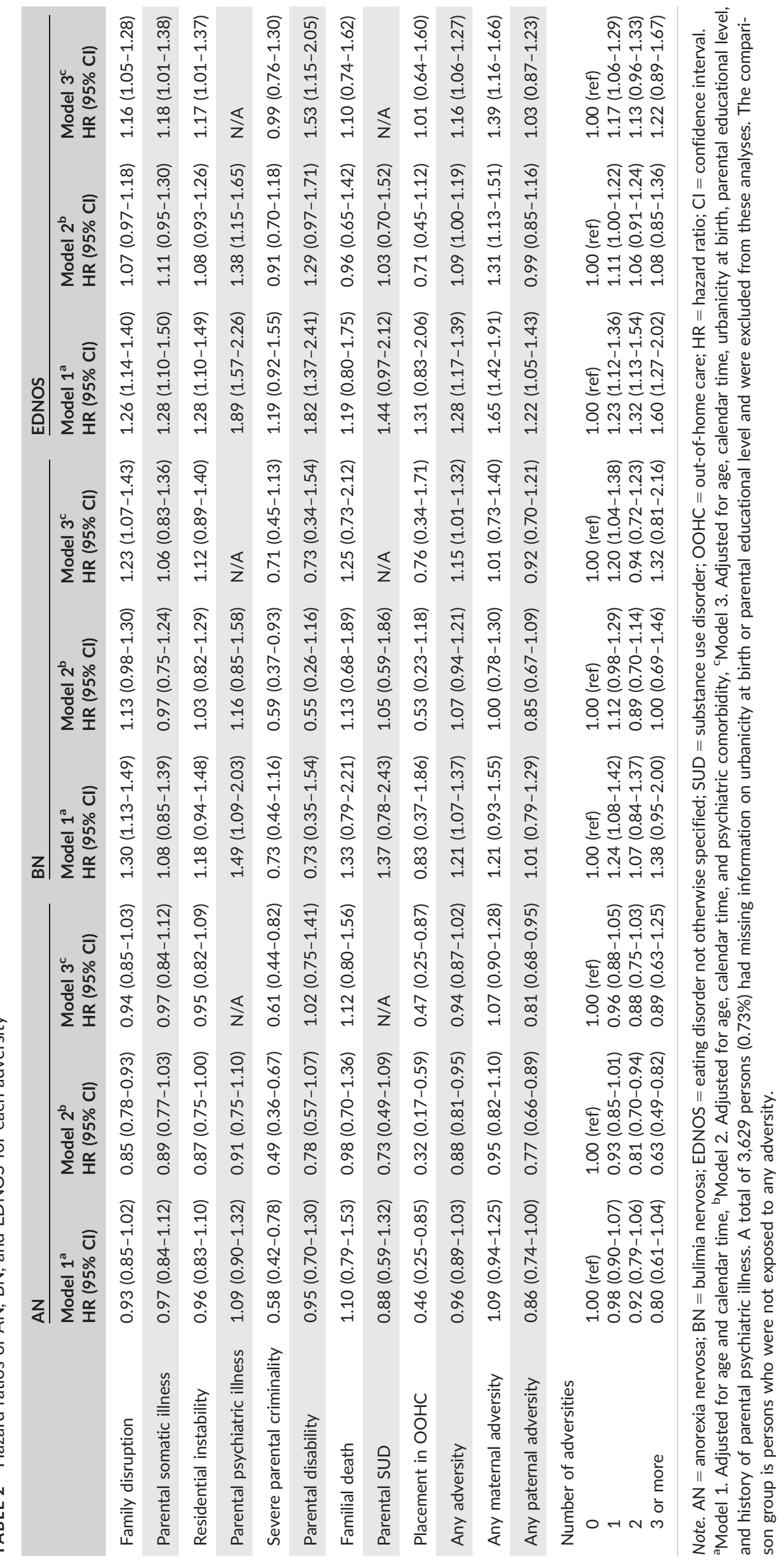



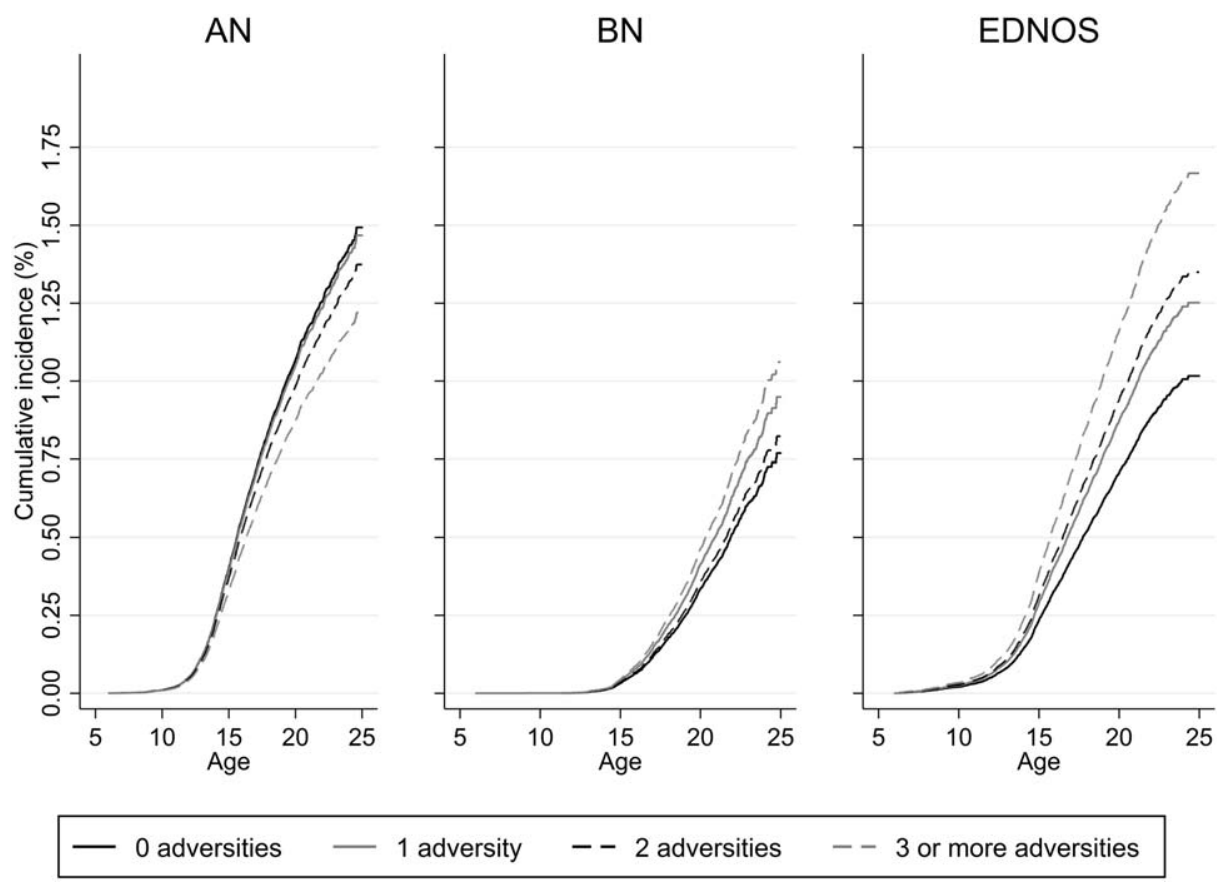

FIGURE 2 Cumulative incidence of AN, BN, and EDNOS by number of adversities experienced. Cumulative incidences were calculated using competing-risks regression with death as the competing event. $\mathrm{AN}=$ anorexia nervosa, $\mathrm{BN}=$ bulimia nervosa, $\mathrm{EDNOS}=$ eating disorder not otherwise specified

\subsection{2 | Bulimia nervosa}

Family disruption, parental psychiatric illness, and any adversity were all significantly associated with increased risk of BN in model 1 with increases in $\mathrm{BN}$ rates of up to $49 \%$, observed for parental psychiatric illness. The associations remained significantly elevated in model 3 . In model 2 , only severe parental criminality was significantly associated with decreased risk of BN. Further, we observed an increasing linear trend for number of adversities, with more adversities associated with increased risk of $\mathrm{BN}$ [change in HR per adversity: 1.11 (1.02-1.20), data not shown].

\subsubsection{Eating disorder not otherwise specified}

In model 1, several types of adversities were significantly associated with increased risk of EDNOS, with increases in rates ranging from $26 \%$ for family disruption to $89 \%$ for parental psychiatric illness. Any adversity, any maternal adversity, and any paternal adversity were also significantly associated with EDNOS. Only the estimates for parental psychiatric illness, any adversity, and any maternal adversity remained significant in model 2, whereas most remained significant in model 3. There was strong evidence of a significant linear trend for number of adversities and increasing risk of EDNOS [change in HR per adversity: 1.17 (1.11-1.24), data not shown].

\subsection{4 | Sensitivity analyses}

Only including the first eating disorder diagnosis ever received in the case definition did little to change the results. The estimates for all three eating disorders are very similar to those presented in Table 2, but for AN they were mostly slightly decreased, and for BN and EDNOS mostly slightly increased (data not shown).

\subsection{5 | Other psychiatric disorders}

To determine the extent to which patterns of adversities seen in eating disorders differ from those observed in individuals with other psychiatric disorders, we conducted parallel analyses with MDD, anxiety disorders, and OCD. All types of adversities were significantly associated with increased risk of MDD in model 1 [with increases in rates ranging from $54 \%$ for parental somatic illness to $157 \%$ for parental psychiatric illness]. All but familial death were significantly associated with increased risk of anxiety disorder [with increases in rates ranging from $51 \%$ for parental somatic illness and residential instability to $211 \%$ for parental SUD]. Most adversities were also significantly associated with increased risk of OCD with increases in rates ranging from $23 \%$ for any paternal adversity to $87 \%$ for parental psychiatric illness. For all three comparison disorders, adjustments in models 2 and 3 did little to change the results and there was evidence of increasing linear trends for number of adversities (data not shown).

\subsection{Cumulative incidences}

Figure 2 shows the cumulative incidences of AN, BN, and EDNOS from age 6 until age 25 for persons who were exposed to 0,1 , 2, or 3 or more adversities between ages 0 and 5. For AN, the cumulative incidence was highest for persons with no adversities and decreased with increasing number of adversities. For BN and EDNOS, the lowest cumulative incidence was observed for persons with no adversities, and it was increased for persons with one or more adversities. 


\section{4 | DISCUSSION}

In general, we found that some childhood adversities (i.e., any paternal adversity, severe parental criminality, and placement in $\mathrm{OOHC}$ ) were associated with a decreased risk of AN, but most adversities were not found to be associated with AN. Conversely, we found that BN and, in particular EDNOS, were more similar to other psychiatric disorders [both in the literature (Björkenstam et al., 2016) and in our comparison groups] and tended to be positively associated with exposure to adversities in early childhood. Adjustment for psychiatric comorbidity further enhanced the inverse associations between early adversities and $\mathrm{AN}$, whereas it somewhat attenuated estimates for BN and EDNOS, but a pattern of elevated risks remained. That $\mathrm{AN}$ stands out as different from both $\mathrm{BN}$ and EDNOS and from the other psychiatric disorders in this regard could suggest alternate risk pathways to the illness. Indeed, single-nucleotide-polymorphism (SNP)-based genetic correlations have revealed intriguing divergences in patterns of correlations with AN relative to other psychiatric and somatic traits and disorders. Although AN is clearly genetically correlated with other psychiatric traits, unlike other psychiatric disorders, AN also appears to have strong correlations with a variety of metabolic and anthropometric parameters, possibly suggesting differences in its genetic etiology (Anttila et al., 2016; BulikSullivan et al., 2015; Duncan et al., 2017; Hinney et al., 2016; ScottVan Zeeland et al., 2014). Although comparable SNP-based correlations are not yet available for BN and EDNOS, the fact that AN has a different genetic and environmental risk profile encourages deeper exploration.

The panel of adversities included in this study was inspired by the study by Dahl et al. (2017) on severe unipolar depressive disorder. For comparison, we kept the same panel of adversities regardless of whether they were significantly associated with eating disorders. We found little evidence supporting associations between childhood adversities and AN, which is in agreement with several studies that have also found no evidence for an effect of parental death (Fairburn et al., 1999; Su et al., 2016) or family disruption (Ahrén et al., 2012, 2013; Björkenstam et al., 2016; Stuart, Laraia, Ballenger, \& Lydiard, 1990) on risk for AN. We found that any adversity related to the father was associated with a decreased risk of AN, which was mainly driven by paternal criminality. To our knowledge, the most comprehensive study to date evaluating the associations between childhood adversities and eating disorders is a Swedish register-based study by Björkenstam et al. (2016) including 107,704 persons born in Stockholm. Among the various adversities examined, only household living on public assistance during childhood, a proxy measure for relative poverty, was significantly associated with decreased risk of pooled eating disorders. They found no evidence for an association between severe parental criminality and eating disorders, but this could be due to their collapsing of all eating disorders into one group and that they started follow-up at age 15 , when a large proportion of persons with eating disorder have already been diagnosed for the first time (Zerwas et al., 2015). We found no evidence of an association between any of the eating disorders and residential instability, which is in accordance with the results of Mok et al. (2016). However, Fairburn et al. (1999) did find that exposure to residential instability as well as parental psychiatric illness and family disruption did increase risk of AN compared to healthy controls without any eating disorders. They also found a dose-response pattern of increasing risk of AN with multiple adversities, which was not evident in our data. These conflicting results could be due to differences in study designs, that is, prospective cohort versus case-control, and the resulting differences in recall bias.

Any adversity was associated with increased risk of BN. We found that exposure to parental psychiatric illness and family disruption in particular increased the risk of BN, which is in accordance with studies by Boumann and Yates (1994) and Webster and Palmer (2000). We observed a positive but nonsignificant effect of familial death, which is in agreement with studies by Stuart et al. (1990), who reported the same for household deaths, and Su et al. (2016) who reported that parental death before age 10 significantly increased risk of BN. The fact that the study by Su et al., which was based on both Danish and Swedish register data, found a significant effect while we did not could be attributed to statistical power issues.

To our knowledge this is the first study to explore the association between various childhood adversities and EDNOS in particular. Su et al. (2016) studied the effect of postnatal loss of parent on the risk of mixed eating disorders (including EDNOS, and overeating and vomiting associated with other psychological disturbances) and found no evidence of an association. This is in accordance with our result on familial death.

\section{1 | Strengths and limitations}

In Denmark, hospital-based treatment is free of charge, but socioeconomic factors still influence the probability of seeking treatment, and register information for the current study did not include primary care contacts. It is very likely that defining psychiatric and somatic illness using registers captures only the most severe cases and results could change, were all detected cases at all points of care to be included. The same can be said for other adversities that we studied; only parents who have been convicted were included in the severe parental criminality adversity and only parents who have been assigned disability pension by the municipality were included in the parental disability adversity. Family disruption was based on whether the child shared an address with both parents and we could not take into account whether the child had regular contact with both parents or not. Placement in $\mathrm{OOHC}$ also only included families with marked dysfunction. All adversity measures were dichotomized based on available register information. This fails to take into account differences in the impact actually experienced by the child, which would be possible to capture in an interview- or questionnaire-based study.

One of the most widely studied environmental risk factors for eating disorders is childhood abuse (Caslini et al., 2016; Racine \& Wildes, 2015; Rayworth, Wise, \& Harlow, 2004; Smolak \& Murnen, 2002; Webster \& Palmer, 2000; Welch \& Fairburn, 1996; Wonderlich, Brewerton, Jocic, Dansky, \& Abbott, 1997). As previously mentioned, we originally intended to include childhood abuse (combined sexual, emotional, and physical abuse captured by contacts to hospitals), but 
unfortunately found that we had insufficient data to do so without compromising Danish legislation regarding personally identifiable information.

In this study, the largest case group was individuals diagnosed with AN, followed by EDNOS and BN. This distribution differs from other studies, where EDNOS comprises the largest group and AN the smallest (Fairburn et al., 2007). Our study only included cases diagnosed with an eating disorder in a hospital, thereby overlooking those diagnosed in primary care only, those with sub-threshold symptoms, and those not seeking treatment (Swanson \& Field, 2016). It is very likely that the inclusion of all of these, if possible, would change the case distribution.

This study only included women, as the incidence of hospitaldiagnosed eating disorders is low in males $(<10 \%$ of the incidence for women-please see Zerwas et al. (2015), which is similar to the present study in design, for details on incidences of diagnosed eating disorders in Danish men), and combined with the relatively rare exposures we were unable to calculate reliable estimates for men.

All adversities were only included for ages 0-5 years, that is, before the start of follow-up. This was done to limit issues regarding reverse causality. It is possible that the stress of a child suffering from severe psychiatric illness such as an eating disorder could influence the occurrence of some of the included adversities, for example, family disruption. By only including adversities experienced in this relatively short, early stage of life, we fail to take into account the impact of later and possibly very important experiences. Even so, we did find several significant associations between these early adversities and later psychiatric outcomes in this relatively young cohort, including for MDD, which generally occurs later in life compared to eating disorders (Pedersen et al., 2014), and for OCD, which in this study was similar to AN and EDNOS in number of cases.

To our knowledge, this is the largest study to date examining the associations between multiple types of early childhood adversity and risk of eating disorders in women. Our study included all women born in Denmark between 1989 and 2007 and was conducted using national registers. This made it possible to follow individuals for up to 19 years from their 6th birthday. Use of register-based information eliminates loss to follow-up, recall and reporting bias. The Danish registers are considered almost complete regarding information on vital and residential information, treatment in hospitals, public financial support, criminal convictions, and placement in $\mathrm{OOHC}$. The large cohort size made it possible to examine the associations between relatively uncommon exposures and outcomes, but also to obtain significant results even when the effect sizes are relatively small. However, our results do suggest that, unlike other psychiatric disorders, AN seems to be either unassociated or inversely associated with exposure to adversities in early childhood. Conversely, early childhood adversities seem to be associated with increased risks of both $\mathrm{BN}$ and in particular EDNOS, similar to what is observed for other psychiatric illnesses. Our findings support that experiences in early childhood impact mental health and disordered eating in adolescence and early adulthood, but also that AN is different from other eating disorders and psychiatric illnesses.

\section{ACKNOWLEDGMENTS}

The work was supported by the Lundbeck Foundation Initiative for Integrative Psychiatric Research (iPSYCH) and funded by the Anorexia Nervosa Genetics Initiative (ANGI), an initiative of the Klarman Family Foundation. Dr. Bulik acknowledges funding from the Swedish Research Council (VR Dnr: 538-2013-8864).

\section{CONFLICT OF INTEREST}

Dr. Bulik is a grant recipient from Shire and member of a Shire scientific advisory board. No other authors have a declaration of interest to disclose.

\section{ORCID}

Janne Tidselbak Larsen MSc (D) http://orcid.org/0000-0002-0385-1017

\section{REFERENCES}

Ahrén, J. C., Chiesa, F., Klinteberg, B., \& Koupil, I. (2012). Psychosocial determinants and family background in anorexia nervosauresults from the Stockholm birth cohort study. International Journal of Eating Disorders, 45(3), 362-369. https://doi.org/10.1002/eat.20953

Ahrén, J. C., Chiesa, F., Koupil, I., Magnusson, C., Dalman, C., \& Goodman, A. (2013). We are family-parents, siblings, and eating disorders in a prospective total-population study of 250,000 Swedish males and females. International Journal of Eating Disorders, 46(7), 693-700. https://doi.org/10.1002/eat.22146

Anttila, V., Bulik-Sullivan, B., Finucane, H. K., Bras, J., Duncan, L., EscottPrice, V., ... Neale, B. (2016). Analysis of shared heritability in common disorders of the brain. bioRxiv, https://doi.org/10.1101/048991

Arcelus, J., Mitchell, A. J., Wales, J., \& Nielsen, S. (2011). Mortality rates in patients with anorexia nervosa and other eating disorders. Archives of General Psychiatry, 68(7), 724-731. https://doi.org/10.1001/archgenpsychiatry.2011.74

Björkenstam, E., Burström, B., Vinnerljung, B., \& Kosidou, K. (2016). Childhood adversity and psychiatric disorder in young adulthood: An analysis of 107,704 Swedes. Journal of Psychiatric Research, 77, 6775. https://doi.org/10.1016/j.jpsychires.2016.02.018

Boumann, C. E., \& Yates, W. R. (1994). Risk factors for bulimia nervosa: A controlled study of parental psychiatric illness and divorce. Addictive Behaviors, 19(6), 667-675. https://doi.org/10.1016/0306-4603(94)90021-3

Bulik-Sullivan, B., Finucane, H. K., Anttila, V., Gusev, A., Day, F. R., Loh, P. R., ... Neale, B. M. (2015). An atlas of genetic correlations across human diseases and traits. Nature Genetics, 47(11), 1236-1241. https://doi.org/10.1038/ng.3406

Caslini, M., Bartoli, F., Crocamo, C., Dakanalis, A., Clerici, M., \& Carra, G. (2016). Disentangling the association between child abuse and eating disorders: A systematic review and meta-analysis. Psychosomatic Medicine, 78(1), 79-90. https://doi.org/10.1097/PSY.0000000000000233

Dahl, S. K., Larsen, J. T., Petersen, L., Ubbesen, M. B., Mortensen, P. B., MunkOlsen, T., \& Musliner, K. L. (2017). Early adversity and risk for moderate to severe unipolar depressive disorder in adolescence and adulthood: A register-based study of 978,647 individuals. Journal of Affective Disorder, 214, 122-129. https://doi.org/10.1016/j.jad.2017.03.014

Duncan, L., Yilmaz, Z., Gaspar, H., Walters, R., Goldstein, J., Anttila, V., ... Bulik, C. M. (2017). Significant locus and metabolic genetic correlations revealed in genome-wide association study of anorexia nervosa. American Journal of Psychiatry. https://doi.org/10.1176/appi.ajp. 2017.16121402 
Fairburn, C. G., Cooper, Z., Bohn, K., O'connor, M. E., Doll, H. A., \& Palmer, R. L. (2007). The severity and status of eating disorder NOS: Implications for DSM-V. Behaviour Research \& Therapy, 45(8), 17051715. https://doi.org/10.1016/j.brat.2007.01.010

Fairburn, C. G., Cooper, Z., Doll, H. A., \& Welch, S. L. (1999). Risk factors for anorexia nervosa: Three integrated case-control comparisons. Archives of General Psychiatry, 56(5), 468-476. https://doi.org/10. 1001/archpsyc.56.5.468

Field, A. E., Sonneville, K. R., Micali, N., Crosby, R. D., Swanson, S. A., Laird, N. M., ... Horton, N. J. (2012). Prospective association of common eating disorders and adverse outcomes. Pediatrics, 130(2), e289-e295. https://doi.org/10.1542/peds.2011-3663

Hinney, A., Kesselmeier, M., Jall, S., Volckmar, A. L., Focker, M., Antel, J., ... Hebebrand, J. (2016). Evidence for three genetic loci involved in both anorexia nervosa risk and variation of body mass index. Molecular Psychiatry. https://doi.org/10.1038/mp.2016.126

Kyvsgaard, B. (2002). The criminal career. The Danish longitudinal study. Cambridge: Cambridge University Press.

Laursen, T. M., Munk-Olsen, T., Nordentoft, M., \& Mortensen, P. B. (2007). A comparison of selected risk factors for unipolar depressive disorder, bipolar affective disorder, schizoaffective disorder, and schizophrenia from a Danish population-based cohort. Journal of Clinical Psychiatry, 68(11), 1673-1681. https://doi.org/10.4088/JCP. v68n1106

Lynge, E., Sandegaard, J. L., \& Rebolj, M. (2011). The Danish National Patient Register. Scandinavian Journal of Public Health, 39, 30-33. https://doi.org/10.1177/1403494811401482

Mok, P. L., Webb, R. T., Appleby, L., \& Pedersen, C. B. (2016). Full spectrum of mental disorders linked with childhood residential mobility. Journal of Psychiatric Research, 78, 57-64. https://doi.org/10.1016/j. jpsychires.2016.03.011

Mors, O., Perto, G. P., \& Mortensen, P. B. (2011). The Danish Psychiatric Central Research Register. Scandinavian Journal of Public Health, 39, 54-57. https://doi.org/10.1177/1403494810395825

Pedersen, C. B. (2011). The Danish Civil Registration System. Scandinavian Journal of Public Health, 39, 22-25. https://doi.org/10.1177/ 1403494810387965

Pedersen, C. B., Mors, O., Bertelsen, A., Waltoft, B. L., Agerbo, E., McGrath, J. J., ... Eaton, W. W. (2014). A comprehensive nationwide study of the incidence rate and lifetime risk for treated mental disorders. JAMA Psychiatry, 71(5), 573-581. https://doi.org/10.1001/ jamapsychiatry.2014.16

Petersson, F., Baadsgaard, M., \& Thygesen, L. C. (2011). Danish registers on personal labour market affiliation. Scandinavian Journal of Public Health, 39, 95-98. https://doi.org/10.1177/1403494811408483

Racine, S. E., \& Wildes, J. E. (2015). Emotion dysregulation and anorexia nervosa: An exploration of the role of childhood abuse. The International Journal of Eating Disorders, 48(1), 55-58. https://doi.org/10. 1002/eat.22364

Rayworth, B. B., Wise, L. A., \& Harlow, B. L. (2004). Childhood abuse and risk of eating disorders in women. Epidemiology (Cambridge, Massachusetts), 15(3), 271-278. https://doi.org/10.1097/01.ede. 0000120047.07140.9d

Scott-Van Zeeland, A. A., Bloss, C. S., Tewhey, R., Bansal, V., Torkamani, A., Libiger, O., ... Schork, N. J. (2014). Evidence for the role of EPHX2 gene variants in anorexia nervosa. Molecular Psychiatry, 19(6), 724-732. https://doi.org/10.1038/mp.2013.91
Smolak, L., \& Murnen, S. K. (2002). A meta-analytic examination of the relationship between child sexual abuse and eating disorders. International Journal of Eating Disorders, 31(2), 136-150. https://doi.org/10. 1002/eat.10008

Stuart, G. W., Laraia, M. T., Ballenger, J. C., \& Lydiard, R. B. (1990). Early family experiences of women with bulimia and depression. Archives of Psychiatric Nursing, 4(1), 43-52. https://doi.org/10.1016/S08839417(05)80010-X

Su, X., Liang, H., Yuan, W., Olsen, J., Cnattingius, S., \& Li, J. (2016). Prenatal and early life stress and risk of eating disorders in adolescent girls and young women. European Child \& Adolescent Psychiatry, 25 (11), 1245-1253. https://doi.org/10.1007/s00787-016-0848-z

Swanson, S. A., \& Field, A. E. (2016). Commentary: Considerations for the use of registry data to study adolescent eating disorders. International Journal of Epidemiology, 45(2), 488-490. https://doi.org/10. 1093/ije/dyw084

Thygesen, S. K., Christiansen, C. F., Christensen, S., Lash, T. L., \& Sørensen, H. T. (2011). The predictive value of ICD-10 diagnostic coding used to assess Charlson comorbidity index conditions in the population-based Danish National Registry of Patients. BMC Medical Research Methodology, 11. https://doi.org/10.1186/1471-2288-11-83

Vassos, E., Agerbo, E., Mors, O., \& Pedersen, C. B. (2016). Urban-rural differences in incidence rates of psychiatric disorders in Denmark. British Journal of Psychiatry: The Journal of Mental Science, 208(5), 435-440. https://doi.org/10.1192/bjp.bp.114.161091

Webster, J. J., \& Palmer, R. L. (2000). The childhood and family background of women with clinical eating disorders: A comparison with women with major depression and women without psychiatric disorder. Psychological Medicine, 30(1), 53-60. https://doi.org/10.1017/ S0033291799001440

Welch, S. L., \& Fairburn, C. G. (1996). Childhood sexual and physical abuse as risk factors for the development of bulimia nervosa: A community-based case control study. Child Abuse \& Neglect, 20(7), 633-642. https://doi.org/10.1016/0145-2134(96)00051-8

Wonderlich, S. A., Brewerton, T. D., Jocic, Z., Dansky, B. S., \& Abbott, D. W. (1997). Relationship of childhood sexual abuse and eating disorders. Journal of the American Academy of Child and Adolescent Psychiatry, 36 (8), 1107-1115. https://doi.org/10.1097/00004583-19970800000018

Zerwas, S., Larsen, J. T., Petersen, L., Thornton, L. M., Mortensen, P. B., \& Bulik, C. M. (2015). The incidence of eating disorders in a Danish register study: Associations with suicide risk and mortality. Journal of Psychiatric Research, 65, 16-22. https://doi.org/10.1016/j.jpsychires. 2015.03.003

\section{SUPPORTING INFORMATION}

Additional Supporting Information may be found online in the supporting information tab for this article.

How to cite this article: Larsen JT, Munk-Olsen T, Bulik CM, et al. Early childhood adversities and risk of eating disorders in women: A Danish register-based cohort study. Int J Eat Disord. 2017;50:1404-1412. https://doi.org/10.1002/eat.22798 